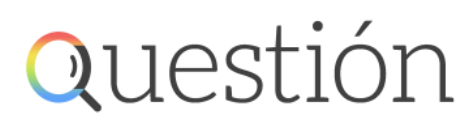

Periodismo / Comunicación ISSN 1669-6581
- Av. $44 \mathrm{~N}^{\circ} 676,1^{\circ}$ piso

CP 1900 - La Plata - Argentina

(www.perio.unlp.edu.ar/question

Las del campo. Testimonios de la enseñanza rural en época de pandemia

Daniela López - Natalia Ragusa

https://doi.org/10.24215/16696581e388

\title{
Las del campo
}

Testimonios de la enseñanza rural en época de pandemia.

\author{
The ones in the countryside \\ Testimonies of rural teaching in times of pandemic.
}

\begin{abstract}
Daniela López
danielalopezz@gmail.com

Es periodista de ciencia, egresada de la Facultad de Periodismo y Comunicación Social de la Universidad Nacional de

La Plata, con maestría en Geografía por la Universidad Nacional Autónoma de México (UNAM).

Escribe historias de ciencia para medios mexicanos e internacionales. Ha dirigido producciones audiovisuales y producido reportajes sonoros para proyectos de comunicación de la ciencia. En el ámbito institucional ha sido coordinadora de vinculación del Fondo para la Comunicación y Educación Ambiental y dirigido el área de difusión en la Unidad de Investigación sobre Representaciones Culturales y Sociales de la UNAM en Morelia. Es miembro de la Red Mexicana de Periodistas de Ciencia y la Red Argentina de Periodismo Científico. maestranda en Gestión y Evaluación de la Educación por la Universidad Nacional de Tres de Febrero. Ha coordinado equipos jurisdiccionales vinculados a la enseñanza de la comunicación en los niveles secundario y superior de la provincia de Buenos Aires y se ha desempeñado como capacitadora en programas educativos nacionales y provinciales en temáticas de educación sexual integral y comunicación y TIC.
\end{abstract}

\section{Palabras claves}

Educación - Pueblos rurales - Covid19 - Mujeres - Enseñanza - Territorio

\section{Resumen}

Las actividades de enseñanza en medio de la pandemia mundial declarada por la Organización Mundial de la Salud en marzo de este año no cesaron en ningún momento en Argentina. Las clases siguieron en cada lugar y todos los niveles y modalidades, como parte de la política educativa nacional y provincial. Este trabajo rescata (y reconoce) la labor de las docentes e invita a los y las lectoras a escuchar los testimonios de maestras rurales de diferentes puntos de la Provincia de Buenos Aires. Es una oportunidad para comprender el quehacer y el sentir del mundo de la enseñanza en el interior de la provincia. 


\section{Keywords}

Educación - Pueblos rurales - Covid19 - Mujeres - Enseñanza - Territorio

\section{Summary}

Teaching activities amid the global pandemic declared by the World Health Organization in March this year did not cease at any time in Argentina. The classes followed at each place and all levels and modalities, as part of national and provincial education policy. This work rescues (and recognizes) the work of teachers and invites readers to listen to the testimonies of rural teachers from different points of the Province of Buenos Aires. It is an opportunity to understand the work and feeling of the world of teaching within the province.

"El tema de que de un día para el otro no pudimos asistir más a la escuela es el cambio más drástico. Porque después todos fuimos buscando la forma y la manera de comunicarnos. Con los nenes que viven en el pueblo y tienen conectividad fue mucho más fácil, con los que viven en el campo y por ahí la conectividad es diferente llevó un poquito más de tiempo o nos comunicamos un día sí un día no, pero el vínculo nunca lo perdimos", dice Verónica Soledad Errasqui de 39 años, directora y docente de la Escuela Primaria №11 de Ordoqui en el partido de Carlos Casares.

1 Testimonio. El tema de que. Verónica: https://ar.ivoox.com/es/51719129

En el ámbito rural bonaerense existen 1.154 escuelas primarias donde estudian 48.304 estudiantes, lo que representa el $2.8 \%$ de los y las alumnas matriculadas en el nivel. Hoy, los y las docentes y estudiantes de estas escuelas llevan adelante las actividades educativas en medio del contexto generado por Covid19.

La Dirección General de Cultura y Educación desde el inicio estableció criterios y recursos para la enseñanza y la evaluación de las prácticas educativas, apuntadas a fortalecer el vínculo pedagógico y garantizar el derecho a la educación aún en tiempos de contingenciai.

Este trabajo busca dar testimonio de la labor que realizan las docentes rurales en diferentes lugares de la provincia de Buenos Aires. No es un análisis de discurso, tampoco se trata de un 
artículo académico, es más bien el deseo de contar, nombrar y poder escuchar a quienes resisten desde el territorio.

Continua Verónica: "En realidad no es que no tienen nada de conectividad, sino que en el campo hay señal en tal horario entonces yo sé que en ese horario los chicos van a tener conectividad y los llamo o en ese horario me puedo comunicar con la mamá para decirles cuál es la actividad. La realidad es que le hemos ido buscando la vuelta".

2 Testimonio. Conectividad. Vero: https://ar.ivoox.com/es/51719148

Los y las ocho alumnas de Carolina Olivera, docente rural de la vieja estación Calfucurá, Partido de Mar Chiquita, viven otra realidad. La conexión a internet es nula o casi, sólo tienen acceso mediante datos del celular y la maestra recorre entre 30 y 40 kilómetros para dejar las actividades.

"Dejo en cada tranquera la bolsita con las actividades y los padres sabiendo voy yo ese día me dejan la bolsita con la devolución de actividades que fueron dadas con anterioridad", comenta la maestra de esta escuela que no tiene agua potable, ni luz eléctrica.

3 Testimonio. Los que sí. Carolina: https://ar.ivoox.com/es/51719175

Dice el antropólogo Hugo Ratier (2009) que en la provincia de Buenos Aires transcurren dos geografías, la que reflejan los mapas viales y las que están a un costado: "campos abiertos que se alargan hacia un horizonte lejano y monótono, con ganados pastando, sembradíos, alguna casa aislada o el casco de la afortunada estancia que quedó sobre la ruta". En ese costado están las escuelas rurales.

"Una escuela rural es un centro social en donde hay todo tipo de eventos: cumpleaños, almuerzos, donde se comparten charlas y experiencias, mateadas, es lo que la escuela le ofrece al campo. Si bien el campo de por sí mantiene un aislamiento social, la escuela vendría a ser como el centro social", señala Carolina.

4 Testimonio. Una escuela rural. Carolina: https://ar.ivoox.com/es/51719241

Y agrega, "Otro de los puntos en donde yo pongo más énfasis es que la escuela debe estar abierta siempre, para lo que sea, es un centro social de encuentro para poder hacer talleres, almuerzos, sin ir más lejos hoy se hizo una vacunación de la gripe A en la escuelita". 
5 Testimonio. Otro de los puntos. Carolina: https://ar.ivoox.com/es/51719260

Algo parecido pasa en Ordoqui. En este pueblo donde hoy viven 174 personas, pero que en la década del 30 llegaron a ser más de 1.800 habitantes (Vesco, 2017), Verónica cuenta que al ser pocos todos están en casi todas las instituciones, incluso la escuela.

"Todos estamos para todos. Por ejemplo, cuando hay las fiestas de fin de año de la escuela, pedimos autorización y las hacemos en el club. Las hacemos en un horario donde pueda ir la mayor parte de la población posible, porque si no son tíos, son abuelos y sino son vecinos que también forman parte de la comunidad", señala.

6 Testimonio. Todos estamos. Vero: https://ar.ivoox.com/es/51719284

De las 434 localidades bonaerenses (López, 2017) con menos de 2.000 habitantes concretamente 170 (Urquijo, 2017) lidian hoy con el problema del despoblamiento. Algunas de las principales causas que contribuyen a esta realidad son viejas deudas no saldadas del cierre de los ramales ferroviarios allá por finales de la década del 70 . El tren, para la mayoría de los pueblos, era el único medio de comunicación con la ciudad y su levantamiento implicó desvincular estos lugares con otros pueblos y ciudades vecinas. Para algunos estudiosos, esta etapa es el inicio de problemáticas devenidas por el aislamiento rural. (García, 2009; López, 2011; Urquijo,2017).

Laura Josende es unidocente de la escuela № 20 Martín Fierro ubicada a 10 kilómetros de la ruta provincial № 29 y a 60 kilómetros de Ciudad de Balcarce. Cuenta que es habitual que los días de lluvia sus alumnos, alumnas y ella no puedan llegar a la escuela. Si bien la pandemia y el aislamiento social obligatorio generaron incertidumbre en la comunidad educativa, en ese momento en el que no hubo tiempo para analizar, Laura recurrió a lo que ya estaba pensado, planificado y que le permitía de alguna manera sostener el vínculo pedagógico y la enseñanza cuando el encuentro en la escuela no estaba garantizado.

"Hay que adaptarse a los días de lluvia, a los caminos de tierra que están inaccesibles para llegar, por lo tanto, los días de lluvia es muy difícil poder acceder y tener la clase como siempre. Es una escuela que está muy adaptada a lo que tenemos en la actualidad. Nosotros el plan de contingencia lo preparamos desde el inicio. En febrero ya lo estábamos armando y ya sabemos cómo actuar en caso de que no podamos concurrir a la escuela o que los alumnos no puedan 
concurrir (...) Es lo que realmente hoy estamos usando, el plan de contingencia o continuidad pedagógica como le estamos diciendo hoy con esto del Covid19", dice Laura.

7 Testimonio Hay que adaptarse Laura: https://ar.ivoox.com/es/51719290

En este juego entre lo planificado y lo emergente se modificó la dinámica de la escuela, de los vínculos y de las prácticas docentes, que como explica Achilli (2001) implican "un conjunto de actividades, interacciones, relaciones que configuran el campo laboral del sujeto maestro 0 profesor en determinadas condiciones institucionales y sociohistóricas. La práctica docente puede implicar actividades que van desde las 'planificaciones' del trabajo áulico a las actividades de asistencias alimenticias, de salud, legales, de colaboración con documentación de los alumnos u otras".

"Hay mucha contención en la llamada. Ellos lo necesitan y uno está a disponibilidad para lo que sea, tanto en la parte afectiva o para algo que ellos necesitan: una mercadería o un médico, lo que sea, uno está ahí atento. Con las nenas hay mucho vínculo, hay una relación de mucho cariño y en este momento nos estamos extrañando muchísimo", dice Laura.

8 Testimonio Hay mucha contención Laura: https://ar.ivoox.com/es/51719305

Verónica, aún en el marco del desconcierto abre lugar a reflexionar sobre el encuentro y la contención afectiva: "Tenemos familias que reciben los bolsones de alimentos entonces es una oportunidad para verlos, con todas las precauciones que tenemos que tener, el uso de barbijos y todo. Es un momento también de preguntarles cómo están, qué necesitan. Porque la realidad es que a todos nos afecta de una manera u otra. Todos los días es un día más y hay que seguir trabajando, seguir buscándole la manera. Y entonces cada oportunidad es un montón para mí, para verlos, para saber cómo están", comparte.

9 Testimonio Tenemos familias Verónica: https://ar.ivoox.com/es/51719317

El ensayo y la búsqueda de formas que permitan mantener el encuentro y enseñar a la distancia, para transitar el aislamiento en momentos de pandemia, es una constante en los relatos de las docentes.

"Yo creo que tenemos un rol fundamental en esto de estar, acompañarlos, escucharlos, que ellos sientan que son importantes para nosotros que están y que los escuchamos. No es, bueno la 
seño llamó, mandó la actividad y ya está ¿no? Sino retribuir esa actividad con una retroalimentación o con una felicitación, ya sea un audio, un sticker, una corrección: felicitaciones, excelente trabajo, bravo. Que se genere este ida y vuelta para que también tenga un sentido", dice Verónica.

10 Testimonios Yo creo que tenemos Verónica: https://ar.ivoox.com/es/51719335

"Entretelones me entero que yo mando una consigna por un audio y hay una mamá que no lo puede bajar, entonces hay una nena del grupo que ella le pasa todo por escrito lo que yo mando en el audio para que el otro compañerito no pierda. $\mathrm{O}$, por ejemplo, hay una cuenta que no se entendió, entonces se mandan entre ellos, aparte, ayudas", cuenta Carolina y pone de manifiesto la solidaridad entre los y las propias compañeras.

11 Testimonios Por entretelones Carolina: https://ar.ivoox.com/es/51719351

Y agrega, "sostengo y sigo sosteniendo que lo importante es no perder el vínculo con ellos, mantenernos siempre comunicados, darles la confianza de que esto va a pasar, que se puede volver a retomar todo otra vez y que aquellas cosas que quedan en duda se vuelven a retomar".

12 Testimonio Sostengo Carolina: https://ar.ivoox.com/es/51719383

Laura y Carolina cuentan que armaron proyectos junto a docentes de otras escuelas rurales, con recursos pensados y diseñados para sus alumnos y alumnas. Por su parte, Verónica refirió a las "ventajas" de la organización de la enseñanza en pluriaños, que aún en pandemia, se han sostenido.

“(...) si lo ves desde otro punto de vista es una ventaja esto de trabajar con chicos de diferentes edades. Por ejemplo, (...) trabajo con nenes que llegan a la escuela y todavía no saben escribir y chicos de tercer grado que ya están autónomos ¿no? y por ahí hay unas personas lo podrían ver como una desventaja y yo que trabajo con ellos, así hace años, lo veo como una ventaja, sí, porque el trabajo en equipo se fomenta mucho más y se trabaja de otra manera porque además son grupos muy pequeños".

13 Testimonio Más allá de la situación: https://ar.ivoox.com/es/51719407 
Verónica no puede más, la escuela del pueblo es su vida y también es su historia: allí creció, egresó y cuando tuvo la oportunidad regresó a enseñar, y eso es lo que más desea en este momento: "Lo primero que espero es que sea pronto, no sé cuánto vamos a tardar en que esto se pueda dar. Uno lo espera con ansia, con eso de querer verlos a todos juntos. Se extraña un montón, yo vivo súper cerca de la escuela y no escuchar el ruido de la campana... esa nostalgia. Es muy ansiado por todos el regreso, tanto por nosotros, como por los chicos".

14 Testimonio. Yo espero. Vero: https://ar.ivoox.com/es/51719427

Los geógrafos culturales dicen que para hablar de lugar tiene que haber un grupo de personas que lo identifique como suyo, que se oriente dentro de él (Claval en Fernández y Urquijo, 2012). La comunidad educativa en los espacios rurales es eso, la pertenencia a un lugar que transgrede los planes de estudios, que hace suyo el proceso de aprendizaje sin importar en qué campo del conocimiento hay que registrar esa página. Por eso, en tiempos de pandemia las comunidades educativas rurales saben que la única forma de resistir es creando estrategias, aceptando el destino y por sobre todas las cosas sabiéndose unidos más allá del aislamiento.

\section{Referencias bibliográficas}

Achilli, Elena (2001) Investigación y formación docente, Laborde, Argentina.

Brumat, R. (2011). Maestros rurales: condiciones de trabajo, formación docente y práctica cotidiana. Revista Iberoamericana de Educación OEI, 55 (4), 1-10.

Dirección General de Cultura y Educación. El estado de la escuela. Datos y estadísticas 2019. Consulta en línea: http://www.abc.gob.ar/sites/default/files/el_estado_de_la_escuela_2019.pdf

Dirección General de Cultura y Educación, (2020) Continuemos estudiando. Sitio web: https://continuemosestudiando.abc.gob.ar/

Fernández, F. y Urquijo, P. (2012). Corografía y escala local. Enfoques desde la geografía cultural. Capítulo I. CIGA. UNAM. México.

García, L. (2009). Territorio e Identidad: ¿ “pueblos fantasmas" o te- rritorios con futuro?: Aportes desde la gestión cultural. FLACSO Argentina.b

López, D. (2011). Un almanaque, un pueblo. Identidad y memoria colectiva en el Almanaque La Niña pueblo rural, Tesis de licenciatura en Comunicación Social. Facultad de Periodismo y Comunicación Social. Universidad Nacional de La Plata. Argentina. 
López, D. (2017) Arraigo y memoria desde la geografía cultural. Caso La Niña, Pueblos Rural. Argentina, Tesis de Maestría en Geografía, Universidad Nacional Autónoma de México. Ratier, H. et al. (2004). Poblados bonaerenses. Vida y milagro, La Colmena, Buenos Aires. Terigi, F., (2008) Organización de la enseñanza en los plurigrados de las escuelas rurales. Tesis de Maestría. Facultad Latinoamericana de Ciencias Sociales (FLACSO), Argentina.

Urquijo Torres, P.S. (2007) Pequeñas localidades rurales. Reapropiación territorial en Argentina y México, Universidad Nacional Autónoma de México: CIGA, Morelia, 222 pp.

Vesco, L. (2017). Pueblos Fantasmas. La Argentina que desaparece. La Nación. Consulta en linea: https://www.lanacion.com.ar/sociedad/pueblos-fantasma-la-argentina-que-desaparecenid1992347

\section{Notas}

i La creación del portal https://continuemosestudiando.abc.gob.ar/ con recursos y actividades para todos los niveles educativos. Recomendaciones sobre herramientas y software, convivencia, uso de las redes sociales y violencia de género. 